\title{
La mangue en Afrique de l'Ouest francophone : variétés et composition variétale des vergers
}

\author{
Jean-Yves ReY ${ }^{a *}$, Thierno Mamadou DIALlo ${ }^{b}$, Henri VANNIĖRE ${ }^{a}$, Christian DIDIER ${ }^{a}$, Sidiki KéıtA ${ }^{c}$, Morodjan SANGARÉc
}

a Cirad, département Flhor, TA 50/PS4, Bd de la Lironde, 34398 Montpellier Cedex 5, France

Jean-yves.rey@cirad.fr

b IER, URG, BP 30, Bamako, Mali

c Irag, CRA Bordo, BP 352 Kankan, Guinée
* Correspondance et tirés à part

Reçu le 16 avril 2004 Accepté le 24 mai 2004

Fruits, 2004, vol. 59, p. 191-208 (C) 2004 Cirad/EDP Sciences All rights reserved

DOI: 10.1051/fruits:2004018

RESUMEN ESPAÑOL, p. 208
The mango in French-speaking West Africa: varieties and varietal composition of the orchards.

Abstract - Introduction. The mango tree is one of the most widespread fruit trees in West Africa. A history of its introduction into that area was recently published. To follow upon this document, the presented analysis was devoted to the principal varieties that are cultivated there today and to the impact of the historical and commercial factors on the varietal composition of the orchards. Some definitions. In precondition to an inventory of the varieties, certain terms making it possible to characterise them were specified (precocity, monoembryony and polyembryony) and some information on the origin of the Floridian varieties was provided. Principal varieties cultivated in West Africa. Four mango variety categories were distinguished: varieties of local or polyembryonic mangos (mangots and Number One), first monoembryonic varieties propagated by grafting (Amélie, Julie, Sabot, Djibelor and Cuisse Madame), and the Floridian varieties, also monoembryonic and propagated by grafting, introduced later and used either for export (Kent, Keitt, Palmer, Zill, Valencia, Smith, Irwin and Haden), or for the regional markets (Brooks, Davis-Haden, Miami Late, Springfels, Beverly, Eldon and Ruby). Each variety was described, like its farming characteristics and its outlets. The composition of the grafted mango tree orchards. This composition is influenced by the historical evolution and the purpose of the fruits that can be eaten, sold on the local, national or subregional market, exported toward the international market or processed in artisanal or industrial units. This orchard composition was specified for Guinea, Mali, Burkina Faso, Côte d'Ivoire, Senegal and Togo.

Francophone Africa / Mangifera indica / introduced varieties / agronomic characters / precocity / fruits / phenotypes

\section{La mangue en Afrique de l'Ouest francophone : variétés et composition variétale des vergers.}

Résumé - Introduction. Le manguier est l'un des arbres fruitiers les plus répandus en Afrique de l'Ouest. Un historique de son introduction dans la zone a été publié récemment. Pour faire suite à ce document, l'analyse présentée a été consacrée aux principales variétés qui y sont cultivées aujourd'hui et à l'impact des facteurs historiques et commerciaux sur la composition variétale des vergers. Quelques définitions. En préalable à un inventaire des variétés, certains termes permettant de les caractériser ont été précisés (précocité, monoembryonie et polyembryonie) et quelques informations sur l'origine des variétés floridiennes ont été fournies. Les principales variétés cultivées en Afrique de l'Ouest. Quatre catégories ont été distinguées les variétés de mangues locales ou polyembryonnées (mangots, mangue du Cameroun), les premières variétés monoembryonnées propagées par greffage (Amélie, Julie, Sabot, Djibelor, Cuisse Madame), les variétés floridiennes, également monoembryonnées et propagées par greffage, introduites plus tardivement et utilisées soit pour l'exportation (Kent, Keitt, Palmer, Zill, Valencia, Smith, Irwin, Haden), soit pour les marchés régionaux (Brooks, Davis-Haden, Miami Late, Springfels, Beverly, Eldon, Ruby). Chaque variété a été décrite, de même ses caractéristiques culturales et ses débouchés. La composition des vergers de manguiers greffés. La composition des vergers greffés est influencée par l'évolution historique et la destination des fruits qui peuvent être autoconsommés, vendus sur le marché local, national ou sous-régional, exportés sur le marché international ou transformés dans des unités artisanales ou industrielles. Cette composition a été précisée pour la Guinée, le Mali, le Burkina-Faso, la Côte d’Ivoire, le Sénégal et le Togo.

Afrique francophone / Mangifera indica / variété introduite / caractère agronomique / précocité / fruits / phénotype 


\section{Introduction}

Selon l'historique déjà publié retraçant la diffusion des manguiers en Afrique de l'Ouest [1], les premiers manguiers plantés dans cette zone ont été les mangots à fruits fibreux, polyembryonnés. Introduits au cours du $\mathrm{XIX}^{\mathrm{e}}$ siècle, ils ont connu un fort développement durant la seconde moitié de ce siècle. Les variétés monoembryonnées greffées ont été implantées après 1890. Dans un premier temps, seules Amélie et, à un degré bien moindre, Divine et Julie ont été diffusées de manière notable. Ce n'est qu'à partir de 1960 et plus encore après 1980 que les variétés floridiennes, introduites en 1949 sur la station de Foulaya, près de Kindia, en Guinée, ont connu un fort développement en Afrique de l'Ouest.

L'étude de l'évolution des exportations intercontinentales [1] a montré qu'elles avaient débuté à la fin des années 1960. Dominées de 1970 à 1990 par le Mali alors suivi du Burkina Faso, elles ont été ensuite principalement alimentées par la Côte d'Ivoire qui, avec le développement des expéditions maritimes, a connu une forte hausse de ses exportations pendant la décennie suivante. Plus récemment, le Sénégal a également beaucoup augmenté ses envois.

Au cours de ces périodes, la variété Amélie a composé l'essentiel des premières exportations. Par la suite, elle a été largement remplacée par les variétés floridiennes (Kent, Keitt, Palmer, Valencia, etc.) [1].

L'étude présentée fait suite à l'historique rapidement évoqué précédemment. Elle s'est intéressée aux principales variétés de manguiers cultivées en Afrique de l'Ouest et à l'impact des facteurs historiques et commerciaux sur la composition variétale des vergers. Certaines notions comme la précocité, la mono et la polyembryonie, et quelques informations sur l'origine des variétés floridiennes, qui, avec Amélie, composent la quasi-totalité du verger greffé ouest-africain, ont été précisées en préalable pour une meilleure compréhension de l'ensemble.

\section{Quelques définitions}

\subsection{La précocité}

Le terme " précoce ", appliqué aux espèces fruitières, peut avoir plusieurs sens. Il peut qualifier soit un arbre qui fructifie rapidement après sa plantation, soit une variété (ou espèce) apte à produire ses fruits avant les autres, au cours de la saison. Dans notre présentation, nous avons utilisé les termes "précoce, précocité "(et leurs antonymes " tardif, tardiveté ") dans ce dernier sens, en réservant l'expression "mise à fruit rapide " pour l'arbre qui fructifie jeune.

Dans les zones de savanes sèches, lieu de production des mangues d'exportation, il existe un gradient des périodes de fructification. Les zones les plus précoces sont situées au sud de Korhogo (Dikodougou, Tafiré) en Côte d'Ivoire et les plus tardives sont localisées plus au nord et, dans une moindre mesure, vers l'ouest. Ainsi, les mangues des Niayes du Sénégal ou celles des zones les plus septentrionales (basse vallée du Sénégal) sont les plus tardives. Cette évolution est due essentiellement à l'allongement de la période froide.

Par ailleurs, la récolte des premiers fruits ne doit pas être confondue avec la pointe de production de la variété au sein d'une même zone. En ville, les arbres plantés dans les cours d'habitation subissent de nombreuses agressions (ligatures par des câbles, écorçage, coups de machettes, enfoncement de pointes) et sont régulièrement enfumés lors de la préparation des repas sur les feux de bois. Or, l'enfumage est une technique utilisée en Asie pour déclencher la floraison des arbres [2]. Cela pourrait expliquer que les vieux manguiers implantés en milieu urbain portent plus de fruits hors saison que les arbres jeunes des vergers ruraux et que de petites productions d'Amélie apparaissent à Bamako dès le mois de février alors que la pointe de production de cette variété se situe en avril-mai. Pour plus de cohérence, dans la suite de notre document, la date de production de la variété sera définie en fonction de la pointe de récolte. 
Au sein d'une zone déterminée, la période de production d'une variété dépend des conditions particulières de la parcelle : microclimat, sol, antécédents de l'arbre, etc. Sur la station de recherche fruitière du Centre national de recherche agronomique (CNRA) de Korhogo au nord de la Côte d'Ivoire, étendue sur 40 ha, la production entre parcelles d'une même variété peut être décalée de près d'un mois.

\subsection{La monoembryonie et la polyembryonie}

Les embryons zygotiques (sexués) proviennent d'une fécondation : le gamète femelle, ou oosphère, à n chromosomes, est fécondé par le gamète mâle, ou noyau spermatique (n chromosomes). À l'issue de cette fécondation, l'œuf ou zygote possède $2 \times$ n chromosomes. Chez les plantes hétérozygotes (non fixées), l'embryon, issu de recombinaisons génétiques lors du processus de méiose et de fécondation, a un génome différent de celui de la mère et de ses frères et sours.

À l'opposé, les embryons apogamiques proviennent de cellules somatiques diploïdes (à 2n chromosomes), le plus souvent originaires du nucelle de l'ovule, d'où leur dénomination d' "embryons nucellaires". Étant issus de la multiplication d'une telle cellule somatique n'ayant subi aucun processus de méiose et de fécondation, ces embryons ont le même potentiel génétique que la plante-mère. Ce mécanisme permet d'obtenir, par semis, des arbres identiques au plant mère, même chez des espèces hétérozygotes. À l'exception de rares cas de polyplö̈die de l'embryon ou de caractères chimériques chez la mère, les plantes issues de semis d'embryons somatiques sont donc génétiquement équivalentes à celles obtenues par multiplication végétative. Les phénotypes s'en différencient toutefois par une longue phase juvénile, raccourcie par la multiplication végétative d'organes adultes.

Chez le manguier, les graines des variétés monoembryonnées contiennent un seul embryon zygotique. Les noyaux de variétés polyembryonnées possèdent un (ou aucun) embryon zygotique et un ou plusieurs embryons somatiques. En pollinisation libre, le pourcentage de noyaux contenant des embryons zygotiques est de $20 \%$ pour Turpentine et de $22 \%$ pour la variété $13-1$, tandis qu'il tombe à $13 \%$ pour Turpentine si ces deux cultivars sont placés en cage d'isolement [3]. Certaines variétés, comme Carabao, Pico et Cambodiana, ne posséderaient que des embryons nucellaires [4].

Généralement, les variétés polyembryonnées sont plus productives. La persistance des embryons nucellaires favorise la nouaison [5]. À l'inverse, chez les variétés monoembryonnées, il est rare que des fruits parthénocarpiques se développent à la suite de l'avortement de l'embryon zygotique. Plus les conditions de milieu sont difficiles pendant la période de floraison, plus les variétés polyembryonnées sont avantagées par rapport aux variétés monoembryonnées. C'est le cas dans les zones de savanes sèches, où la basse hygrométrie de la saison d'harmattan ne favorise pas toujours une bonne pollinisation.

\subsection{L'origine génétique des variétés floridiennes}

Les manguiers considérés comme originaires de la région indo-birmane [1] auraient été diffusés dans deux directions : vers la Malaisie et l'Asie du Sud-Est, en donnant des variétés polyembryonnées, et en direction du golfe du Bengale et de l'Inde en donnant les variétés monoembryonnées [4]. De fait, la majorité des mangues indiennes sont monoembryonnées. Les rares variétés polyembryonnées présentes sur la côte ouest de l'Inde auraient été introduites à Goa par les Portugais, à partir de leurs possessions asiatiques, Macao ou Timor [4].

Sur le continent américain, les manguiers ont été introduits depuis ses deux origines primaires. Au XVIII ${ }^{e}$ siècle, les variétés asiatiques, polyembryonnées, ont été plantées en Amérique centrale (Mexique et Panamá) puis dans la Caraibe par les Espagnols qui avaient atteint l'Asie par l'océan Pacifique après avoir contourné l'Amérique par le détroit de Magellan [6]. Les noms des variétés mexicaines comme Manila ou Filipino rappellent cette origine. D'autres manguiers seraient venus de l'est, introduits après des 
relais en Afrique ou dans les îles de l'océan Indien dans un premier temps. Les Portugais auraient planté les premiers manguiers à Bahia, au Brésil, mais "pas avant 1700 " selon Popenoe [6]. En 1782, un navire contenant des plants de manguiers provenant de l'île de France, l'actuelle île Maurice, destinés à Hispaniola (Haïti, Saint-Domingue) fut capturé par des pirates anglais et envoyé à la Jamaïque, où les arbres furent plantés et prospérèrent. Trente-deux ans plus tard, le manguier y était devenu une plante très commune [6].

Aux États-Unis, la première introduction aurait été réalisée près de Miami en 1833, avec des plants du Mexique. Mais ensuite, avec les progrès des moyens de transport, les importations de diverses origines se sont amplifiées [6]. À partir de 1889, des plants monoembryonnés indiens greffés ont été systématiquement introduits en Floride par le United States Department of Agriculture (USDA) et, parmi eux, la variété Mulgoba, dont une graine allait donner naissance à Haden [6, 7]. De nombreux cultivars floridiens, comme Eldon, Smith, Lippens, Tommy Atkins, Zill, Glenn, Springfels, Valencia, descendent de Haden. Keitt serait issu directement de Mulgoba tandis que la variété Irwin proviendrait de Lippens, et Spirit of 76 de semis de Zill. Edwards serait un hybride de Haden et Carabao [7].

Après Mulgoba, Sandersha [6, 7] fut la seconde variété monoembryonnée indienne utilisée comme géniteur en Floride. Brooks et Anderson proviennent de semis de Sandersha, tandis que Kent serait issu de Brooks et que Fascell serait un hybride de Haden et de Brooks [7]. L'appellation Saigon a été attribuée à un groupe d'arbres provenant d'un lot de graines que D. Fairchild avait envoyé à l'USDA à partir de Saigon, aujourd'hui Hô Chi Minh-Ville au Vietnam. Cambodiana provient d'une sélection effectuée au sein de ce groupe d'arbres très hétérogènes quant à leur taille et leur forme, et la variété Florigon serait issue d'un semis de Saigon [6, 7]. Les parents de Palmer et Ruby sont inconnus [7].

Ainsi, la plupart des variétés floridiennes que nous avons plus spécifiquement étudiées descendraient de deux variétés indiennes monoembryonnées.

\section{Les principales variétés cultivées en Afrique de l'Ouest}

Bien que l'introduction des mangues en Afrique de l'Ouest soit récente, les habitants distinguent les " mangues locales " ou mangots, polyembryonnées, reproduites par semis, fibreuses pour la plupart, des " mangues importées ", monoembryonnées, multipliées par greffage. Pour des raisons pratiques, nous reprendrons cette différenciation en classant les variétés de manguiers d'Afrique de l'Ouest en trois groupes principaux : les variétés polyembryonnées, les variétés floridiennes et les autres variétés représentées essentiellement par Amélie.

\subsection{Les mangues locales ou polyembryonnées}

\subsubsection{Mangot Nunkourouni, ou Tête de Chat}

Le mangot Nunkourouni (figure 1), à fibres et polyembryonné, est répandu dans toute l'Afrique de l'Ouest et en Afrique centrale. Dans chaque site, il porte un nom différent qui peut être lié à l'origine de son introduction (Sierra Leone au Sénégal, mangot allemand dans certaines zones du Cameroun), à la zone où il est cultivé (mangue de N'gaoundéré, dans l'Adamaoua au Cameroun) ou à la forme du fruit. Dans les langues d'origine mandingue, la forme du fruit est souvent assimilée aux éléments du visage (bouche, nez, etc.). Cette mangue s'appelle donc Nez Court ou Tête de Chat (Nunkourouni ou Diakuma Koun ou Mankoron Ba). Elle est appelée également Greffeul au Sénégal.

L'arbre est vigoureux, pourvu d'un feuillage vert foncé. Les feuilles sont longues à bords ondulés; l'inflorescence est teintée de rouge. Les arbres adultes sont très productifs. La production est très précoce en saison, ce qui en fait les premières mangues sur le marché.

Le fruit est aplati latéralement, de forme arrondie et pourvu d'une épaule ventrale descendant assez bas. Il existe de nombreux types locaux et la forme des fruits varie selon les zones de production. Leur poids varie de (150 à 300) g. La peau est rouge, 


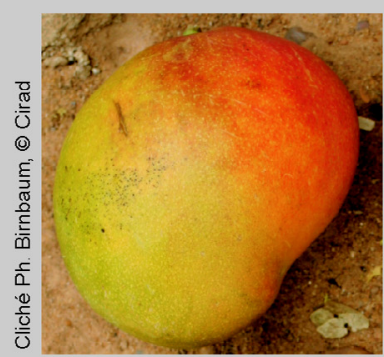

Mangot Nunkourouni ou Tête de chat

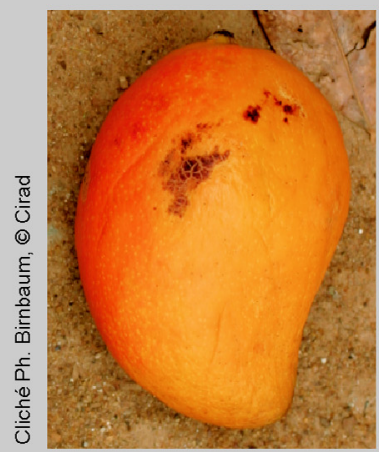

Mangot Dadiani ou Bouche longue

Variété Sabot

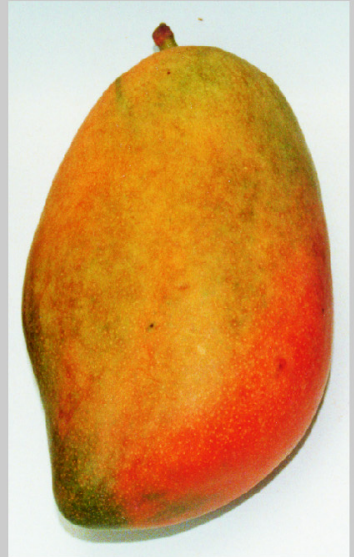

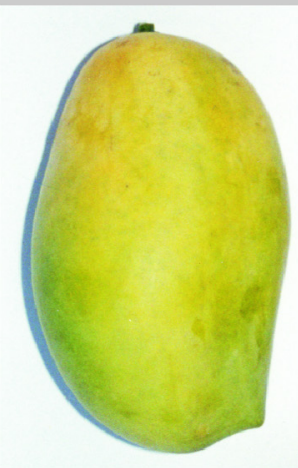

Variétés polyembryonnées propagées par semis

$10 \mathrm{~cm}$

\section{Mangue du littora}

ou Number one ou mangue du Cameroun

Variété Djibelor ou variété $P$ êche

Variété Amélie
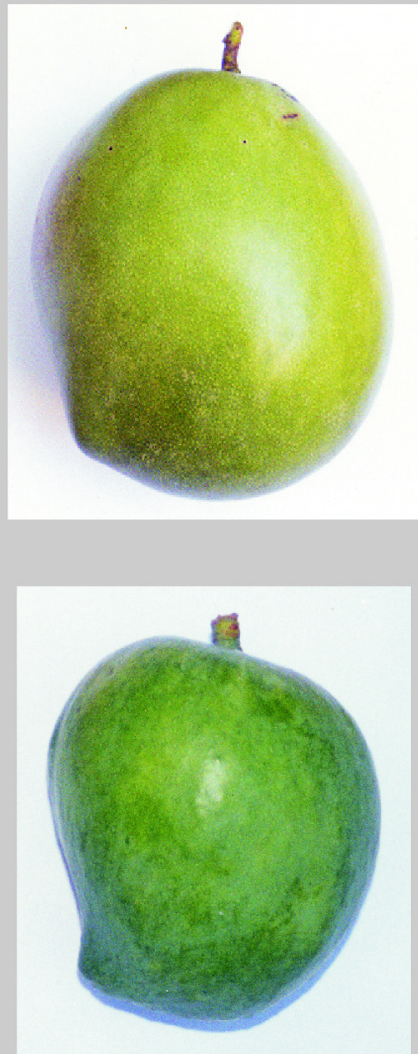

Variétés monoembryonnées propagées par greffage

\section{Figure 1.}

Quelques variétés de mangues polyembryonnées consommées localement et présentation de certaines variétés monoembryonnées parmi les premières qui ont été propagées en Afrique de l'Ouest par greffage. 
jaune ou verte à maturité. La pulpe orange vif, riche en composés volatils [8], est d'un goût très agréable. Les très nombreuses fibres courtes, adhérentes au noyau, ont longtemps nuit à son utilisation industrielle. Toutefois, des procédés modernes comme la technique de "flash-détente " permettent d'utiliser la pulpe sans avoir le désagrément des fibres.

Le nombre d'arbres produisant des mangots Nunkourouni par pays est difficile à évaluer car la plupart des enquêtes sur les manguiers ne prennent pas en compte les arbres de semis considérés comme de faible intérêt économique. Les vergers purs de cette variété sont rares aujourd'hui, mais elle est présente dans tous les lieux de vie : places, cours, marchés, gares routières, bordures de routes et de rues, isolés ou en bosquets dans les champs. Comme elle est utilisée en tant que porte-greffe, il est possible d'observer des pieds isolés dans les vergers quand la greffe échoue ou lorsqu'un rejet a pris l'ascendant sur le greffon. Si une repousse du porte-greffe n'a pas été éliminée, les branches de la variété greffée cohabitent alors avec celles du porte-greffe.

Dans une ville comme Conakry (Guinée), il y a au moins un manguier de cette variété par maison. Entre Conakry et Mamou, distantes d'environ $300 \mathrm{~km}$, ces manguiers bordent la route sur plus de la moitié du parcours. Le manguier Nunkourouni est présent dans toutes les villes de Haute-Guinée, où il constitue l'arbre d'ombrage des avenues et places publiques. Cette variété est présente non seulement dans tous les pays d'Afrique de l'Ouest, mais aussi bien dans les zones humides proches de l'océan que dans les savanes sèches.

La production de mangots Nunkourouni est aussi difficile à évaluer que le nombre de ses arbres car les fruits sont autoconsommés ou commercialisés sur les marchés locaux. Les enfants récoltent les mangots situés en haut de la cime des arbres en grimpant dans les branches ou en les faisant tomber en lançant des cailloux. Les évaluations donnent des rendements moyens de (150 à 200) $\mathrm{kg}$ de fruits par arbre et par an. La production pourrait être évaluée au minimum à $50000 \mathrm{t}$ par pays, mais à beaucoup plus pour des pays comme la Guinée ou le Mali.

\subsubsection{Mangot Dadiani, ou Bouche Longue}

Tout comme le manguier à mangots Nunkourouni, la variété polyembryonnée qui produit le mangot Dadiani (figure 1) est connue sous différentes appellations. L'arbre a un port moins érigé que les manguiers précédents. L'inflorescence est jaune blanchâtre. Le fruit est petit, de (150 à 250) g. Sa peau est vert clair à jaune avec des marbrures roses à rouges à maturité. L'épaule ventrale est courte et l'apex long, d'où les noms de Bouche Longue ou Nez Long (Dadiani, Nundiani). Dans les publications de Ollé et al. [9, 10], elle est désignée sous le nom de Mbengué, ville où elle avait été récoltée pour ces essais. La partie apicale du fruit est droite ou légèrement recourbée, d'où l'appellation "Sabre " qui lui est donnée dans certaines collections. La chair est jaune à orange clair. Les fruits sont sensibles à l'anthracnose. Les fibres sont nombreuses, la pulpe est moins aromatisée et plus fragile que celle de Nunkourouni. La productivité des arbres adultes est très bonne. La production est précoce en saison et coïncide avec celle de Nunkourouni. Bien que largement représentée, cette mangue est toutefois moins répandue que la précédente.

Pour ces deux mangots, la fragilité de la pulpe, la petite taille des joues et les difficultés d'utilisation agroalimentaire de la pulpe fibreuse par les techniques classiques font que ces fruits ont surtout été réservés à l'autoconsommation et aux marchés locaux. Leur abondance en saison de production permet à de larges couches de la population de les consommer à satiété. En Guinée, les habitants préfèrent ces variétés à toutes les autres et les variétés greffées précoces trouvent peu de preneurs.

\subsubsection{Autres mangots et variétés locales}

En dehors des mangots Nunkourouni et Dadiani, il existe d'autres mangots fibreux comme Mangotine (Fumani), à petit fruits de (80 à 100) g et très parfumés, et Mangot Vert, introduits des Antilles à la station de Foulaya (Guinée). Certaines autres variétés peuvent être abondantes à l'échelle d'une région ou d'un pays, comme le petit mangot appelé Séwal au Sénégal. 


\subsubsection{La mangue du Cameroun}

La mangue du Cameroun (figure 1) nécessite une présentation particulière. Introduite au départ dans la région de Douala (Cameroun), cette variété polyembryonnée a été propagée par semis le long du littoral avant d'être largement diffusée vers l'intérieur du pays, à Yaoundé, en zone forestière, puis vers le nord et les pays voisins. Elle est connue sous différents noms dont "Mangue du Littoral ", dans la partie francophone, et "Number One ", en zone anglophone, sont les plus connus. À l'extérieur du Cameroun, elle est appelée Mangue du Cameroun ou Améliorée du Cameroun (à l'origine ce dernier terme englobait cinq clones sélectionnés par de Laroussilhe, qui les avait nommés Ifac 1 à Ifac 5).

Dans plusieurs pays d'Afrique de l'Ouest, cette variété a été diffusée essentiellement par greffage. Consommée localement, elle n'a jamais eu l'importance relative qu'elle possède en Afrique centrale.

L'arbre peut atteindre un fort développement en conditions favorables. Les feuilles sont grandes, à bords ondulés. Les fruits petits, à peau jaune ou verte à maturité, ont une pulpe fragile, peu fibreuse, très sensible au jelly seed autour du noyau. Selon les fruits, la couleur de la pulpe varie d'orange vif à blanchâtre en passant par différentes nuances de jaune. Le goût est assez discret, agréablement parfumé, légèrement acidulé.

L'arbre et le fruit rappellent certaines variétés du Sud-Est asiatique. Son principal intérêt est de fructifier abondamment dans les zones humides. Mais les caractéristiques du fruit (peau verte, fragilité de la pulpe, petit format, etc.) nuisent à sa commercialisation lointaine.

\subsection{Les premières variétés monoembryonnées propagées par greffage}

\subsubsection{Amélie}

La variété Amélie (figure 1), dont on ne connaît pas réellement l'origine, fut introduite des Antilles à la mission catholique de Kita. Est-ce la même variété que la Reine-Amélie dont parle Sébire dans la collection de Thiès
[9] ? Elle est actuellement largement répandue dans toutes les zones de savanes sèches au nord du $9^{\circ}$ de latitude $N$, même si elle se trouve parfois en mélange dans des vergers situés plus au sud, au Togo, par exemple. Cette répartition géographique est liée à sa sensibilité aux maladies cryptogamiques des fleurs et des fruits (oïdium, anthracnose). Son importance relative en savane lui a valu le nom de Greffée en de nombreux lieux où elle fut longtemps la seule variété greffée. Elle est également dénommée Gouverneur, en particulier au Burkina-Faso.

L'arbre a un port caractéristique, très différent de celui des variétés floridiennes. De taille modeste, il présente une frondaison dense en boule. Les branches, très ramifiées, portent de petites feuilles vert clair, plates, à bords réguliers.

Le fruit a une peau verte ou jaune à maturité qui, sur les sols latéritiques, peut prendre une très belle coloration rouge sur la partie exposée au soleil. La chair est d'un bel orange vif. Les taux de sucre et d'acidité sont variables selon les conditions de culture mais généralement inférieurs à ceux des cultivars floridiens [10]. Le goût et l'odeur sont aisément reconnaissables. Selon Ollé et al. [9], les arômes d'Amélie sont particuliers, " étant pratiquement carencés en car-3-ène et possédant (Z)- et (E)-ocimène comme principaux monoterpènes. Ces deux derniers composés volatils ont une odeur chaude, herbacée et florale, tandis que l'odeur du car-3-ène est douce, rappelant le limonène raffiné".

Ces arômes auraient aussi été retrouvés dans de la purée et des fruits mûrs ou verts de mangues Alphonso indiennes ou dans celles du cultivar Jaffna cultivé dans le nord du Sri Lanka [8]. En Afrique de l'Ouest, nous ne connaissons qu'un seul cultivar ayant le même goût qu'Amélie. Il s'agit d'une variété à épiderme d'un rouge carmin très coloré se trouvant en collection au CNRA, à Korhogo, mais pratiquement pas diffusée.

Amélie est la plus précoce des variétés commerciales. La conservation au froid du fruit vert est satisfaisante malgré une certaine sensibilité à la frisure qui provoque une dégradation assez rapide des fruits mûrs. Le manque de coloration rouge de l'épiderme 


$\begin{aligned} & \text { Tableau I. } \\
& \text { Répartition des fleurs femelles et taux de fructification de trois variétés de manguiers } \\
& \text { à la station de Lataha, Korhogo, nord de la Côte d'Ivoire (d'après Briot [11]). }\end{aligned}$
\begin{tabular}{lccc} 
Variété & $\begin{array}{c}\text { \% de fleurs femelles en } \\
\text { position apicale }\end{array}$ & $\begin{array}{c}\text { Moyenne du \% de fleurs } \\
\text { femelles }\end{array}$ & $\begin{array}{c}\text { Taux de fructification } \\
\text { par panicule }(\%)\end{array}$ \\
\hline Amélie & $22,7( \pm 7,9)$ & $11,1( \pm 4,1)$ & 10 \\
Keitt & $65,5( \pm 18,0)$ & $30,1( \pm 8,9)$ & 31 \\
Kent & $32,5( \pm 8,9)$ & $20,5( \pm 6,0)$ & 25
\end{tabular}

de cette variété et sa durée de vie commerciale relativement courte incitent les importateurs européens à lui préférer d'autres cultivars, mais, sa précocité permet de l'exporter jusqu'à l'arrivée des variétés rouges.

La production d'Amélie, qui peut atteindre plusieurs centaines de kilos sur certains arbres, est très faible en moyenne, du fait notamment d'un mauvais taux de fécondation (tableau I). Dans le nord de la Côte d'Ivoire, les rendements moyens en fruits d'exportation de cette variété oscillent entre (1 et 2$) \mathrm{t} \cdot \mathrm{ha}^{-1}$.

\subsubsection{Julie et Sabot}

La variété Julie ${ }^{1}$ d'Afrique de l'Ouest est différente de celle des Antilles françaises, mais elle est proche de la variété Sabot (figure 1). Le port de l'arbre ressemble à celui d'Amélie, mais les feuilles sont ondulées. Le fruit est plat, de forme carrée ou ovale chez Julie, en forme de sabot chez... Sabot. La couleur de l'épiderme varie du vert au marron à maturité avec parfois des taches rouges. La chair, très fragile, d'une couleur jaune foncée à orange, possède un goût musqué très prononcé apprécié des consommateurs locaux, qui ont donné à Julie le nom de Muscat. Le fruit est pratiquement intransportable en raison de sa rapidité de maturation et de la fragilité de sa pulpe. Son aire de répartition est la même que celle d'Amélie, mais à

\footnotetext{
${ }^{1}$ Il existe une Julie Kasowa présente dans les collections de l'Irad (Institut de recherche agricole pour le développement) au Cameroun, qui est diffusée assez largement dans les provinces septentrionales de ce pays sous le nom de Julie, mais qui est différente des mangues de la variété Julie d'Afrique de l'Ouest.
}

part quelques pieds conservés par les planteurs pour leur consommation personnelle, ces variétés sont en voie d'abandon.

\subsubsection{Djibelor}

En Casamance, dans le sud du Sénégal, la mangue de Djibelor également appelée pêche (figure 1) est excellente et sans fibres. Elle est proche d'une variété indienne appelée Paheri (synonymes : Peter Passand, Alphonse Paheri, Païri).

\subsubsection{Cuisse Madame}

Cuisse Madame entrait dans la composition d'un verger établi près de Siguiri, en HauteGuinée, en 1931. Elle produit un gros fruit à épiderme vert. Sa diffusion se limite à la Haute-Guinée.

\subsection{Les variétés floridiennes utilisées pour l'exportation}

Les variétés floridiennes monoembryonnées étant largement répandues dans le monde, nous nous sommes attachés à décrire leurs caractéristiques dans les conditions spécifiques d'Afrique de l'Ouest.

\subsubsection{Kent}

La variété Kent (figure 2), d'origine floridienne, a été introduite à la station de Foulaya, en Guinée, par Py en 1949. Depuis une dizaine d'années, elle supplante la variété Amélie en tant que principale variété d'exportation d'Afrique de l'Ouest. Certaines caractéristiques des fruits sont fortement influencées par les types de sol. Sur la station du CNRA de Lataha, près de Korhogo, d'une superficie de 40 ha, certaines parcelles peuvent fructifier près d'un mois après les 


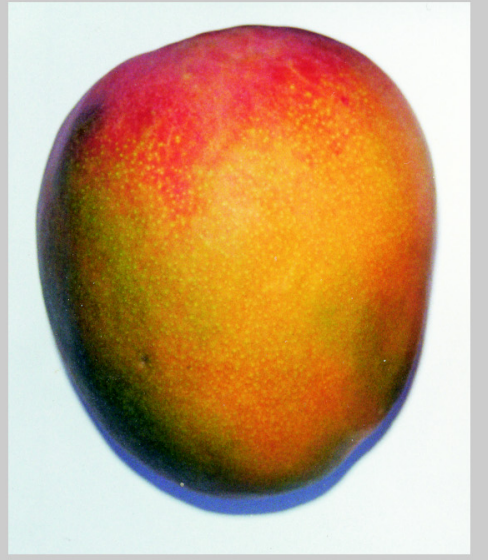

Variété Kent

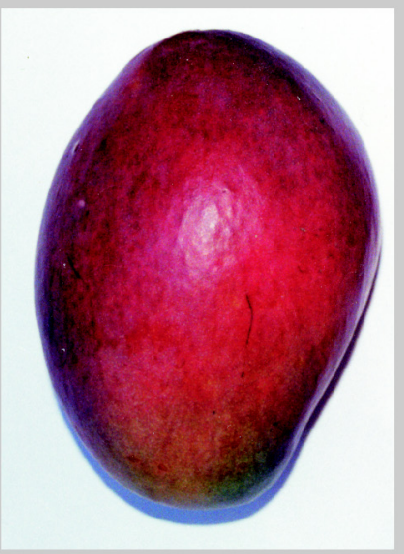

Variété Palmer

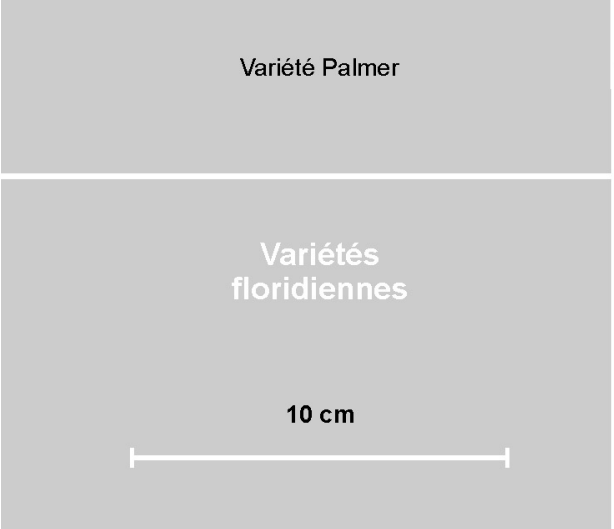

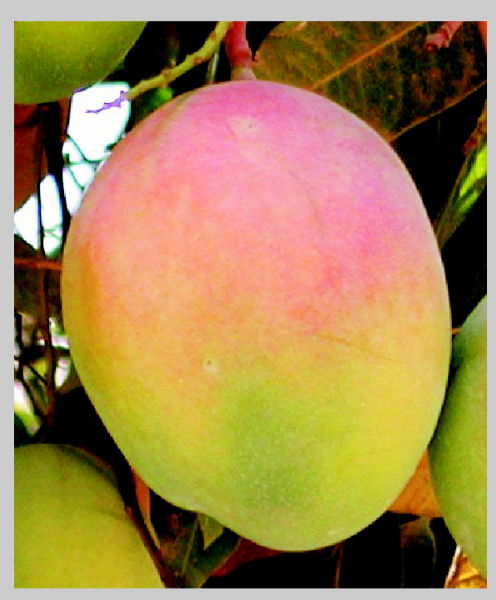

Variété Keitt

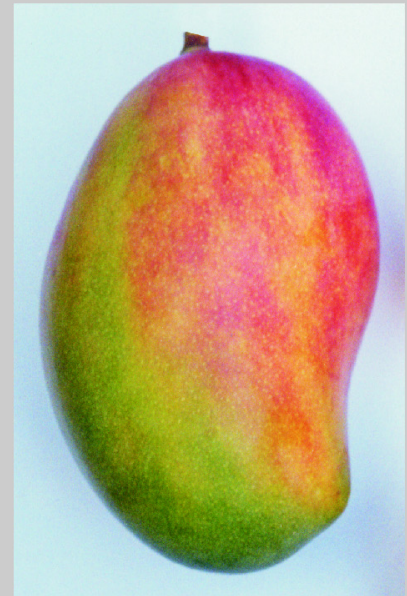

Variété Valencia

Variétés monoembryonnées

utilisées pour l'exportation

\section{Figure 2.}

Présentation de variétés monoembryonnées floridiennes produites en Afrique de l'Ouest et utilisées soit pour l'exportation, soit pour les marchés nationaux ou régionaux. 


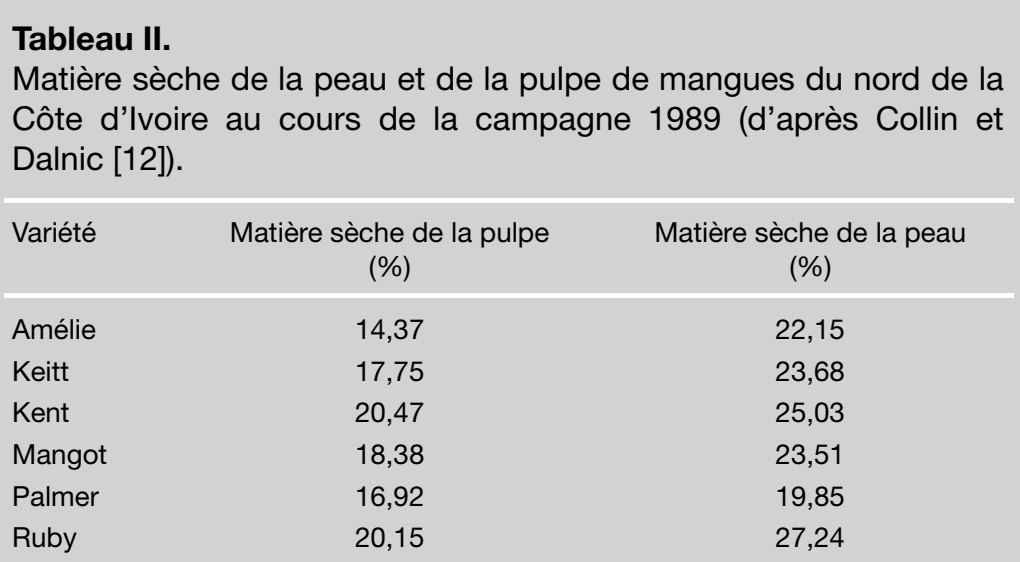

autres. Les fruits sont précoces, fermes et colorés sur les coteaux latéritiques, alors qu'ils peuvent être vert foncé, tardifs et atteints de désordres physiologiques sur les sols de bas-fonds argileux et humides.

Les traditions Sénoufos, ethnie principale du nord de la Côte d'Ivoire, visaient à conserver un équilibre entre les cultures annuelles (cotonniers et plantes vivrières) et les productions pérennes (manguiers, anacardiers et espèces forestières). Les premiers vergers de manguiers ont souvent été relégués sur les collines de graviers latéritiques, impropres aux cultures annuelles, mais aptes à produire des fruits précoces et magnifiques. Ce fait a été l'un des éléments favora- bles au développement des exportations ivoiriennes.

Kent produit entre le 10 avril et le 20 mai dans le sud de la zone d'exportation (Korhogo, Ferké en Côte d'Ivoire), en mai à Sikasso (Mali) et dans l'ouest du BurkinaFaso, à partir du 15 mai autour de Kankan (Guinée), de mai à juillet à Bamako (Mali) et de juillet à septembre dans les Niayes (Sénégal). Certains vergers des environs de Korhogo peuvent être entièrement récoltés à la mi-avril.

La variété Kent se caractérise par une teneur en eau de la pulpe relativement faible, qui pourrait expliquer sa bonne durée de vie commerciale (tableau II). La maturation du fruit est très progressive. L'extrait sec soluble des mangues Kent prêtes à être consommées est élevé et peut atteindre $22^{\circ} \mathrm{Brix}$ dans certains fruits, alors que l'acidité, plus faible à la récolte que chez les autres variétés citées, est plus élevée après maturation (tableau III).

Les analyses de mangues du nord de la Côte d'Ivoire effectuées par Collin et Dalnic confirment ces résultats [12]. En revanche, les analyses réalisées par Ramata Diallo sur des fruits de Guinée-Maritime à maturité indiquent des extraits secs solubles et des acidités inférieurs à ceux de Korhogo pour Kent et Keitt (tableau IV). Ces analyses effectuées en juillet, soit 1,5 mois après le début de la saison des pluies, confirment

Tableau III.

Extrait sec soluble (ESS) et acidité de trois variétés de mangues d'exportation cueillies au stade de maturité export à Korhogo, nord de la Côte d'Ivoire (d'après Briot [11], Guépratte [13] et Moulio [14]).

\begin{tabular}{|c|c|c|c|c|}
\hline \multirow[t]{2}{*}{ Variété* } & \multicolumn{2}{|c|}{ Analyses à la récolte } & \multicolumn{2}{|c|}{ Analyses après maturation } \\
\hline & ESS ( ${ }^{\circ}$ Brix) & $\begin{array}{c}\text { Acidité } \\
\text { (meq pour } 100 \mathrm{~g} \text { de pulpe) }\end{array}$ & ESS ( ${ }^{\circ}$ Brix) & $\begin{array}{c}\text { Acidité } \\
\text { (meq pour } 100 \mathrm{~g} \text { de pulpe) }\end{array}$ \\
\hline Amélie 89 j & $6,8 \pm 0,8$ & $26,8 \pm 3,8$ & $13,9 \pm 1,9$ & $9,0 \pm 3,4$ \\
\hline $\begin{array}{l}\text { Kent } 120 \mathrm{j} \\
\text { (floraison de janvier) }\end{array}$ & $9,4 \pm 1,5$ & $15,6 \pm 5,2$ & $19,9 \pm 2,2$ & $11,2 \pm 2,3$ \\
\hline $\begin{array}{l}\text { Kent } 120 \mathrm{j} \\
\text { (floraison de février) }\end{array}$ & $9,4 \pm 1,0$ & $18,8 \pm 4,8$ & $19,6 \pm 1,4$ & $10,6 \pm 2,1$ \\
\hline Keitt $132 \mathrm{j}$ & $10,5 \pm 1,6$ & $19,4 \pm 4,3$ & $18,5 \pm 1,4$ & $7,9 \pm 1,8$ \\
\hline
\end{tabular}

* Le nombre de jours correspond à l'écart, choisi arbitrairement, entre la floraison de la fleur terminale de la panicule et la récolte. Janvier ou février sont les mois de floraison des inflorescences ayant donné naissance aux fruits. 
que Kent a les extraits secs solubles les plus élevés parmi les variétés analysées. Un certain nombre de fruits (Kent et Keitt) étaient atteints de désordres physiologiques internes.

La taille des fruits de Kent, qui appartiennent essentiellement aux calibres de 7 à 10 (nombre de fruits par carton de $4 \mathrm{~kg}$ ), correspond tout à fait à la demande du marché européen, français en particulier.

Quand les fruits de savane sèche mûrissent, la couleur verte de l'épiderme disparaît en grande partie pour laisser place à un beau jaune. Les fruits mûrs sont alors rouges et jaunes ou rouges, verts et jaunes. Ils sont beaucoup plus attirants lorsqu'ils sont mûrs qu'à leur stade de récolte.

La variété Kent cumule donc beaucoup de qualités commerciales, ce qui explique son succès auprès des importateurs européens : bonne tenue au transport, durée de conservation élevée du fruit mûr maintenu au froid, extrait sec soluble important, belle coloration des fruits produits en conditions adéquates, calibres convenables.

\subsubsection{Keitt}

La variété Keitt (figure 2) a été introduite, comme Kent, par Py à Foulaya, en 1949. Elle est la plus tardive des variétés d'exportation. Bien que certains vergers produisent à partir de la mi-avril près de Korhogo, sa période de récolte s'étale de fin avril à début juin dans le nord de la Côte d'Ivoire. À Sikasso (Mali) et dans l'ouest du Burkina-Faso (Orodara, Banfora), elle produit en mai-juin, et jusqu'en juillet et parfois août. À Bamako (Mali), la récolte s'étale de juin à août et le Sénégal en exporte jusqu'à septembre-octobre.

La durée de maturation des fruits est très longue. Elle peut atteindre 3 semaines à température normale pour des fruits évoluant ensuite normalement. En revanche, les fruits mûrs ont une durée de vie commerciale inférieure à celle de Kent. L'extrait sec soluble est relativement élevé même si les moyennes sont légèrement inférieures à celles de Kent (tableaux III, IV).

La couleur du fruit à la récolte est variable : vert, jaune clair avec ou sans plages d'un rouge cuivré. Toutefois, elle évolue peu au

\begin{tabular}{|c|c|c|}
\hline Variété & $\begin{array}{c}\text { Extrait sec soluble } \\
\left({ }^{\circ} \text { Brix }\right)\end{array}$ & $\begin{array}{c}\text { Acidité } \\
\text { (meq pour } 100 \mathrm{~g} \text { de pulpe) }\end{array}$ \\
\hline Kent & 14,6 & 6,2 \\
\hline Keitt & 13,9 & 3,2 \\
\hline Sabot & 13,7 & 7,4 \\
\hline Lippens & 12,9 & 2,5 \\
\hline Beverly & 12,5 & 10,8 \\
\hline Brooks & 11,4 & 6,6 \\
\hline Irwin & 12,1 & 4,9 \\
\hline
\end{tabular}

cours de la maturation. Les fruits de Keitt, qui sont plus colorés que ceux de Kent lors du conditionnement, sont moins attractifs à maturité.

Le calibre des fruits est aussi variable selon les conditions de milieu. En coteaux gravillonnaires, les fruits sont fermes, colorés et de calibre satisfaisant pour l'exportation (6 à 10), bien qu'une partie notable de la production doive être écartée en raison d'une taille excessive. En revanche, sur sols riches et humides, leur peau reste verte, voire vert foncé (" noir " dans le vocabulaire des récolteurs), et le calibre est trop gros pour l'exportation (6 et moins). Certains fruits peuvent dépasser $1,5 \mathrm{~kg}$. Il arrive aussi que l'on observe des désordres physiologiques, toutefois moins intenses que sur Kent.

Les pédoncules fructifères sont très longs et les branches peu ramifiées et souples. Les fruits sont ainsi exposés à différents aléas : frottements contre le sol ou les branches, dégâts d'insectes, etc. L'épiderme est beaucoup plus sensible aux blessures que celui de Kent. Malgré une bonne fécondation, la production moyenne de fruits d'exportation reste modérée, de (3 à 5) t $\cdot h^{-1}$ dans le nord de la Côte d'Ivoire, ce qui en fait néanmoins la variété la plus productive en fruits d'exportation.

\subsubsection{Palmer}

L'introduction de la variété Palmer (figure 2) en Afrique de l'Ouest est identique à celle des variétés précédentes. C'est une variété 
semi-tardive à tardive selon le lieu. Elle produit plutôt après Kent : en mai et début juin au sud de la zone d'exportation, et de mai à juillet, au nord, dans la région de Bamako. Les arbres sont productifs. C'est l'une des variétés les plus colorées. À l'exception des fruits enfouis à l'intérieur de la frondaison, plus de la moitié du fruit est colorée d'un pourpre sombre. Le reste est vert à la récolte et devient jaune à maturité.

Le calibre des fruits est variable mais globalement plus petit que celui de Kent, avec une forte proportion de mangues de (300 à 450) g. Cette variété est peu sensible aux désordres physiologiques et se comporte bien à la conservation. Son taux de brix est plus élevé que celui d'Amélie : 17,2 ${ }^{\circ}$ Brix pour Palmer contre $13,1^{\circ}$ Brix pour Amélie [10].

La variété Palmer est assez difficile à travailler pour l'exportation. Sa coloration rouge apparaît bien avant la maturité du fruit et elle est trompeuse pour les récolteurs. La sève abondante et brûlante entraîne des brûlures de l'épiderme si l'on ne prend pas de précautions. La mise en cartons des fruits allongés n'est pas aisée : en dehors des petits calibres, il n'est pas possible de disposer les fruits verticalement et, quand ils sont mis à plat, le carton risque de ne pas atteindre le poids minimal.

Cette variété est assez appréciée sur les marchés de l'Europe du Nord, en Belgique en particulier, où les calibres moyens, inférieurs à ceux du marché français, sont appréciés. En France, la mangue Palmer est commercialisée de façon variable selon les négociants mais d'une manière générale elle est moins bien vendue que Kent.

\subsubsection{Zill}

Après avoir été très appréciée à une certaine époque, la variété Zill est peu recherchée actuellement malgré sa très belle coloration à maturité. Elle souffre de défauts majeurs, en particulier son évolution très rapide à l'approche de la maturité entraîne une très courte durée de vie commerciale. Souvent peu productive, elle fournit dans l'ensemble des fruits de petit calibre. Son intérêt est de parvenir à maturité quelques jours avant Kent, ce qui permet d'exporter quelques fruits rouges en période de commercialisation d'Amélie.

\subsubsection{Valencia}

La variété Valencia (figure 2), qui était assez utilisée lors des exportations par avion, a été complètement abandonnée en Côte d'Ivoire avec le développement des exportations par bateau, en raison de sa mauvaise tenue au transport. Elle a, en revanche, été redécouverte par les exportateurs de Bamako expédiant par avion, qui apprécient sa belle coloration. L'arbre a un fort développement végétatif, mais il n'est pas en rapport avec son volume de production. À Bamako, la variété Valencia produit avant Kent.

\subsubsection{Smith}

La variété Smith produisant en saison ou semi-tardive a d'assez bon rendements. Elle est très sensible aux conditions de milieu, tant pour les qualités internes qu'externes. Elle montre souvent des problèmes physiologiques, tels que la surmaturité précoce de la partie apicale ou le développement de racines dans la pulpe. La coloration rouge est importante, mais elle varie d'un vermillon éclatant à un rouge vineux terne. Le calibre peut être satisfaisant (7 à 9) ou trop gros avec une majorité de fruits hors normes. C'est dans la zone de Yagoua, au nord du Cameroun, que l'on peut voir les plus beaux spécimens de cette variété : production abondante, fruits de calibre moyen, belle couleur, chair ferme, etc. Comme la variété Smith produit en même temps que Kent dans la zone actuelle d'exportation, elle ne présente plus grand intérêt.

\subsubsection{Irwin}

Pourvue d'une très belle coloration, la variété Irwin a une forme variable selon les zones de production. Dans la région de Kindia, en Guinée, son fruit a une forme générale ovale alors que, dans le nord de la Côte d'Ivoire, elle prend l'aspect d'un " $\mathrm{S}$ " avec de nombreuses malformations (bosses, excroissances digitées, etc.). Ces anomalies ne sont pas d'origine clonale puisque des greffons prélevés sur des arbres de Kindia 
qui donnaient de beaux fruits ont reproduit les mêmes anomalies à Korhogo. Longtemps principale variété d'exportation de la Guinée-Maritime, le cultivar Irwin a été abandonné, au profit de Keitt, par les exportateurs guinéens qui estiment qu'Irwin mûrit trop rapidement pour le transport par bateau.

\subsubsection{Haden}

La variété Haden a des fruits colorés en jaune, vert et rouge vif à maturité, ce qui en fait la plus belle des variétés d'exportation. Leur chair est sucrée et bonne, malgré la présence de petites fibres. Selon les conditions de milieu, leur taille est moyenne à petite. La production de l'arbre est faible à moyenne selon le sol et les ressources en eau. Ce cultivar, qui aurait pu être largement diffusé, est peu répandu, sans doute pour des raisons historiques - il ne figurait pas dans les premières collections - mais aussi du fait de sa modeste productivité en conditions difficiles et de quelques problèmes physiologiques. Les rares arbres en production sont difficilement utilisés car les exportateurs ne disposent pas de suffisamment de fruits en même temps pour pouvoir confectionner des palettes complètes des différents calibres. De ce fait, les planteurs ne sont pas encouragés à planter davantage. Seuls quelques envois par avion sont réalisés.

\subsection{Les variétés floridiennes utilisées pour les marchés nationaux et régionaux}

\subsubsection{Brooks}

La variété Brooks (figure 2) à épiderme vert, blanchâtre ou jaune à maturité est souvent appelée "Retard " en raison de sa production tardive, de juin à octobre en Côte d'Ivoire. La productivité de l'arbre est très élevée et régulière d'une année à l'autre. Les fruits ont une chair ferme, sans désordres physiologiques, appréciée des consommateurs locaux. Elle est en revanche très sensible aux piqûres de mouches des fruits. Le calibre est régulier avec une majorité de fruits entre (350 et 450) g, ce qui en fait de bons fruits portions. Il ne lui manque que la couleur pour être une excellente variété d'exportation !

\subsubsection{Davis-Haden}

Le cultivar Davis-Haden est appelé Kent Rouge au Mali en raison de la belle coloration de l'épiderme. Les fruits ont un poids moyen variant de (500 à 1 200) g et ils peuvent dépasser $1500 \mathrm{~g}$. Sur de tels fruits la maturité de la chair est très irrégulière. La récolte généralement effectuée à partir de juillet est tardive et les fruits sont très sensibles aux piqûres de mouches et aux maladies fongiques, ce qui entraîne beaucoup de pertes. La taille des fruits et leur sensibilité aux maladies et ennemis rendent difficile l'utilisation de cette variété à l'exportation intercontinentale.

\subsubsection{Miami Late}

La variété Miami Late (figure 2) a une forme rappelant celle de Kent avec toutefois une proportion de surface rouge moins développée. Au Mali, elle est appelée "Souroukou Koun " (Tête de hyène). Les lenticelles sont apparentes et, quand elle est mûre, elle ne présente pas la couleur éclatante de Kent. Les fruits ont une taille régulière et pèsent généralement de (400 à 600) g bien que quelques fruits puissent être exceptionnellement gros (plus de $1 \mathrm{~kg}$ ). La période de production est variable selon les conditions de milieu, mais globalement elle est semitardive à tardive. La production est abondante et régulière. C'est, avec Brooks, l'une des variétés les plus productives, mais c'est aussi l'une des plus sensibles aux piqûres de mouches. Dans les zones humides, elle est attaquée par l'anthracnose. Sa tenue en conservation n'est pas très bonne.

\subsubsection{Springfels}

Springfels est, avec Davis-Haden, le cultivar qui produit les plus gros fruits, ce qui lui a valu le surnom de Papaye, donné par comparaison avec les papayes locales à gros fruits et non par rapport à la variété de papaye Solo. L'épiderme est jaune et rouge terne à maturité. La pulpe contenant de grosses fibres longitudinales, assez douce, est appréciée localement. La production est semi-tardive et peu abondante en général.

\subsubsection{Beverly et Glazier}

Ces deux variétés, introduites originellement à Foulaya comme toutes les mangues 
floridiennes, étaient disposées côte à côte dans la collection implantée sur la station de recherche guinéenne et leurs fruits se ressemblent, ce qui a pu provoquer des confusions par la suite. Si au Mali ou en Guinée ces cultivars sont bien distingués, il n'en est pas de même en Côte d'Ivoire, où la variété connue sous le nom de Beverly est en réalité Glazier, beaucoup moins bonne que la première. Glazier a des taches rouges sur les parties exposées au soleil alors que Beverly est plus claire, voire blanche dans certaines conditions. Les fruits sont moyens à gros, peu attrayants à maturité, sensibles aux piqûres de mouches et semi-tardifs.

\subsubsection{Eldon}

La variété Eldon produit abondamment en saison des fruits jaunes avec une coloration cuivrée plutôt discrète. Le calibre est satisfaisant, de (300 à 450) g, et le goût peu prononcé. Ce cultivar à fruits moyens dans tous les domaines ne présente aucune qualité qui pourrait le distinguer et justifierait des plantations importantes pour le marché local ou l'exportation. Son principal défaut réside dans la sécrétion d'une sève abondante et brûlante qui cause des dégâts au contact de l'épiderme.

\subsubsection{Ruby}

Surnommée " Mademoiselle " dans le nord de la Côte d'Ivoire, la variété Ruby est recherchée par les consommateurs qui apprécient son goût acidulé et parfumé. L'épiderme est fortement coloré en rouge, mais les fruits sont tout petits et pèsent généralement moins de $150 \mathrm{~g}$. Ces mangues sont saines et peu attaquées par les mouches. Même lorsqu'elles sont nombreuses sur l'arbre, le poids récolté reste modeste en raison de la faible taille des fruits. Les producteurs conservent quelques arbres pour l'autoconsommation ou la vente locale.

\section{La composition des vergers de manguiers greffés}

La composition des vergers greffés est influencée par l'évolution historique et la destination des fruits, qui peuvent être soit autoconsommés, soit vendus sur le marché local, national ou sous-régional, soit exportés sur le marché international ou transformés dans des unités artisanales ou industrielles.

L'évolution historique permet de distinguer quatre phases de diffusion des manguiers en Afrique de l'Ouest: l'expansion des mangots de semis, l'établissement des vergers d'Amélie, la diffusion des mangues colorées à partir des collections multilocales et la concentration variétale sur Kent et Keitt sous l'influence des exportateurs. Ces étapes se sont souvent recoupées, ainsi, dans certaines zones, la multiplication des variétés rouges a eu lieu alors que l'expansion des Amélie se poursuivait.

\subsection{La Guinée}

En Guinée, il existe deux grandes régions de production. La Guinée-Maritime est la zone exportatrice de mangue d'Afrique de l'Ouest la plus humide. Elle est plantée essentiellement avec des variétés qui furent introduites à la station de Foulaya (Kindia). Les exportations, qui ne représentent qu'un faible pourcentage de la production, n'ont pas eu d'impact significatif sur les choix variétaux des producteurs, d'autant que les choix des exportateurs ont évolué au cours des dernières années (de Irwin à Keitt).

La Haute-Guinée possède un climat plus chaud et sec que celui de la GuinéeMaritime : il rappelle celui des principales zones productrices des pays voisins (Côte d'Ivoire, Mali, Burkina Faso). Les premiers manguiers greffés ont surtout été des Amélie, plantés dans la zone la plus proche du Mali (Siguiri). L'extension des manguiers s'est ensuite opérée après l'implantation, en 1967, d'une usine de transformation qui produisait de la pulpe de mangue. Cette usine a fonctionné plus ou moins régulièrement jusqu'en 1984 avant d'être fermée officiellement en 1985. Durant son activité, le choix variétal s'était alors porté sur des variétés à gros fruits, productives et tardives pour allonger la période d'approvisionnement de l'usine. Parmi les variétés disponibles en Guinée, Keitt et Miami Late correspondaient alors le mieux à ces critères. 
Après la fermeture de l'usine, les commerçants du marché national ont pris le relais pour écouler la production. Mais comme le marché de la mangue est saturé en début de saison par l'abondante production de mangots, les variétés précoces ou de saison se vendent très mal. La demande est plus forte pour les gros fruits tardifs et sucrés, qui se commercialisent relativement bien dans les régions minières et forestières. Keitt et Miami Late continuent donc à être les variétés les plus plantées. La demande des exportateurs est très faible dans la zone. Il s'agit essentiellement d'agents des exportateurs ivoiriens, qui ne viennent pas en Guinée régulièrement chaque année. La variété Keitt est appréciée par le marché intérieur et non comme variété d'exportation ; c'est ce qui explique que la production de Kent reste modeste alors que celle de Keitt est importante.

La Haute-Guinée est l'une des zones de production qui connaît les plus gros problèmes de mévente d'Afrique de l'Ouest. Cela est dû en partie à l'éloignement de certaines zones de production des grands centres de commercialisation. Dans les autres pays, il est relativement rare de voir des fruits pourrissant sur les arbres, faute d'acheteurs.

\subsection{Le Mali}

Au Mali, les différentes influences historiques et économiques se superposent pour présenter une grande diversité de situations. Autour de Bamako et dans la vallée du Niger située entre la frontière guinéenne et Koulikoro, les premiers vergers greffés avaient été constitués de manguiers Amélie. Cette variété est encore maintenant très nettement majoritaire dans un rayon de $70 \mathrm{~km}$ autour de la capitale malienne.

À partir du début des années 1970, des notables de Bamako développèrent des vergers de prestige. Situés à faible distance de la ville, ces vergers étaient des lieux de détente et de villégiature. Leur production était en grande partie destinée à la consommation de la famille et des amis et les ventes couvraient tout ou partie des frais de cultures. Les plantations constituaient une mise en valeur du terrain permettant d'établir une "lettre d'attribution ", étape importante vers l'obtention du titre foncier. Les planteurs cherchaient alors à avoir une production échelonnée pour étaler au maximum la période de récolte. Bien qu'il soit de bon ton de posséder quelques variétés à très gros fruits, le goût des mangues destinées à l'autoconsommation était plus important que leur aspect. Cependant, pour satisfaire des goûts variés et étaler la production, la plupart de ces vergers étaient de véritables collections.

Plus tard, les besoins des marchés ont entraîné une réduction de la gamme variétale et, aujourd'hui, les planteurs souhaitent davantage rentabiliser leurs plantations qu'il y a 30 ans. Cependant, de nombreux vergers familiaux continuent à être plantés par des citadins exerçant une activité principale non agricole. Aux côtés des vergers paysans, ces vergers de citadins ont amplement contribué à l'approvisionnement en fruits de la capitale malienne et ils ont fourni une partie non négligeable des mangues d'exportation. Dans une phase ultérieure, des vergers purs constitués de Kent et Keitt ont été implantés dans un rayon de moins de $30 \mathrm{~km}$ de la capitale.

Autour de Sikasso, la constitution de certains vergers témoignent également des trois phases de plantation. Les vergers de mangues floridiennes coexistent aux côtés de vergers d'Amélie, mais ils sont composés d'une gamme de variétés beaucoup plus étroite que dans la région de Bamako. Ces variétés sont essentiellement celles qui étaient utilisées pour l'exportation lorsqu'elle se faisait par avion ; cependant, la variété Brooks s'ajoute à elles. Par ailleurs, au sud de Sikasso notamment, il existe d'importants vergers de Kent et Keitt dont les fruits sont destinés à l'exportation par avion ou par bateau.

La zone de Bougouni-Yanfolila a subi de nombreuses influences : celles de la phase d'extension des manguiers Amélie, de la mise en place d'un verger de comportement à Yanfolila, de la proximité de la Guinée et de la Côte d'Ivoire. L'influence guinéenne se traduit par l'abondance des variétés Miami Late et Keitt autour de Yanfolila et entre cette ville et la frontière. Près de la Côte d'Ivoire, de grands vergers de Kent et Keitt ont été implantés. Ces variétés se retrouvent également à proximité de Bougouni. Tous les types de vergers sont présents à Yanfolila. 
Selon une enquête de la délégation régionale de l'agence pour la Promotion des filières agricoles (Aprofa Mali) à Sikasso, citée par le Centre agro-entreprise (CAE) [15] "L'étude d'évaluation du potentiel agricole de la filière de la mangue en $3^{\mathrm{e}}$ Région estime le disponible exportable au niveau de la région de Sikasso pour les trois principales variétés à 48181 t, dont 22233 t pour Amélie (46\%) ; 14076 t pour Kent (29,2\%) ; 11872 t pour Keitt (24,6\%). Avec l'hypothèse que seulement $30 \%$ sont susceptibles d'être exploités pour l'exportation en tenant compte des normes d'exportation et du degré d'enclavement des zones de production, le potentiel d'exportation du Mali pour la $3^{\mathrm{e}}$ Région peut être estimé à $14454 \mathrm{t} "{ }^{2}$. Les mangues non exportées de la $3^{\mathrm{e}}$ Région sont expédiées à Bamako, dans les villes du nord (Ségou, Mopti, Gao) ou vers les pays voisins (Sénégal, Mauritanie).

\subsection{Le Burkina Faso}

Au Burkina Faso, les principaux vergers de manguiers sont situés dans le sud-ouest du pays, à Banfora et Orodara notamment. La phase d'extension de la variété Amélie s'y est prolongée beaucoup plus longtemps que dans les pays voisins. Des vergers de cultivars floridiens variés ont été plantés de façon très modérée. En revanche, les dernières années ont vu une grande extension des Kent et Keitt par la plantation de nouveaux vergers ou le surgreffage.

\subsection{La Côte d'Ivoire}

Dans le nord de la Côte d'Ivoire, les premiers vergers ont été constitués d'Amélie. Puis des vergers de diverses variétés floridiennes se sont développés et leurs fruits ont été utilisés pour les exportations par avion de 1980 à 1992. La demande de Kent et de Keitt, qui a accompagné le développement des exportations par bateau, s'est

\footnotetext{
${ }^{2}$ Au Mali, la $3^{\mathrm{e}}$ Région comprend les villes de Sikasso, Kadiolo, Bougouni, Yanfolila et Koutiala. Elle est bordée au sud par la Côte d'Ivoire, à l'est par le Burkina-Faso et à l'ouest par la Guinée (Mandiana).
}

traduite par l'implantation de nouveaux vergers constitués exclusivement de ces deux variétés et le surgreffage des anciens vergers en Kent et Keitt. En dehors des variétés d'exportation (Kent, Keitt, Amélie, Palmer et quelques Zill), seuls des manguiers de Brooks et divers cultivars conservés par les planteurs pour la consommation familiale sont présents. Cependant, plus de $95 \%$ des arbres greffés appartiennent aux variétés d'exportations.

Dans les zones pluvieuses, les fruits ne sont pas exportés en raison des risques phytosanitaires provoqués par l'humidité. Les fruits destinés au marché local sont assez diversifiés, mais il n'y a pas de grands vergers comme dans les zones sèches.

\subsection{Le Sénégal}

Au Sénégal, les premières productions de variétés greffées, Amélie et Divine, étaient destinées à l'approvisionnement des marchés urbains, Dakar notamment. L'extension des manguiers greffés dans les Niayes a tout d'abord été influencée par les collections réduites introduites auprès de divers organismes ou planteurs privés. Cependant, au cours des dernières années, des surfaces importantes ont été plantées en Kent et Keitt pour satisfaire l'exportation ou le marché national. Les arbres sont arrosés, au moins au cours de leur phase de jeunesse, ce qui entraîne des coûts de production élevés. La demande du grand marché urbain que représente la ville de Dakar contribue à tirer les prix vers le haut. La production de Casamance, composée de diverses variétés, est peu influencée par l'exportation.

\subsection{Le Togo}

Au Togo, la société publique Togofruits avait implanté une collection réduite à partir de greffons venant du Mali. Ce verger comprenait la variété Amélie et divers cultivars floridiens qui ont été diffusés dans le nord du pays mais aussi dans les zones humides. Ainsi, des vergers de manguiers sont rencontrés aux alentours de Kpalimé. Les variétés les plus fréquentes sont celles issue de l'essai variétal de Bamako (Zill, Irwin, Smith, 
Kent, Keitt, Palmer, Valencia, Ruby et Amélie), auxquelles il faut ajouter quelques autres comme Eldon ou Springfels. Les quantités transformées ou exportées sont faibles et l'essentiel de la production est destiné au marché national ou régional.

Dans les autres pays, la production est essentiellement orientée vers le marché national.

\section{Références}

[1] Rey J.Y., Diallo T.M., Vannière H., Didier C., Kéita S., Sangaré M., La mangue en Afrique de l'Ouest francophone, Synthèse historique, Fruits 59 (2004) 121-129.

[2] Singh L.B., The mango, Leonard Hill Books Limited, London, UK, 1960, 439 p.

[3] Degani C., Cohen M., Reuveni O., El-Batsri R., Gazit S., Frequency and characteristics of zygotic seedlings from polyembryonic mango cultivars, determined using isozymes as genetic markers, in Bruce Schaffer (Ed.), Fourth int. mango symp., ISHS, Acta Hortic., no. 341, Wageningen, Netherlands, 1993, pp. 78-85.

[4] Mukherjee S.K., Introduction: botany and importance, in: Litz R.E. (Ed.), The mango: botany, production and uses, Cab Int., Oxon, UK, 1997, pp. 1-19.

[5] Nagasone H.Y., Paull R.E., Mango, in: Tropical fruits, Cab Int., Oxon, UK, 1998, pp. 208238.

[6] Popenoe W., Manual of tropical and subtropical fruits excluding the bananas, coconut, pineapple, citrus fruits, olive and fig, Hafner Press, Div. Macmillan Publ. Co., New York, USA, 1920.
[7] Anonymous, Mangos: guide to mangos in Florida, Campbell R.J. (Ed.), Fairchild Tropical Garden, Miami, USA, 1992.

[8] Sébire A., Les plantes utiles du Sénégal, J.B. Baillière et fils, Paris, France, 1899, pp. 79-80.

[9] Ollé D., Baumes R.L., Bayonove C.L., Lozano Y.F., Sznaper C., Brillouet J.M., Comparison of free and glycosidically linked volatile components from polyembryonnic et monoembryonnic mango (Mangifera indica L.) cultivars, J. Agric. Food Chem. 46 (3) (1998) 10941100.

[10] Ollé D., Lozano Y.F., Brillouet J.M., Isolation and characterisation of soluble polysaccharides and insolubles cell wall material of the pulp from four mango (Mangifera indica L.) cultivars, J. Agric. Food Chem. 44 (1996) 2658-2662.

[11] Briot E., Étude de la physiologie postrécolte de la mangue: projet de fin d'études, UTC de Compiègne, France, 1999.

[12] Collin M.N., Dalnic R., Comparaison de mangues en provenance de Côte d'Ivoire, in: Journ. agrumes/mangues, Irfa, Inra, Montpellier, France, 1991.

[13] Guépratte M., Physiologie postrécolte de la mangue et conservation sous atmosphère modifiée, Mémoire de fin d'étude, École supérieure d'agriculture d'Angers (ESA), 1998, $44 \mathrm{p}$.

[14] Bissardon F., Physiologie postrécolte de la mangue et conservation en froid de la mangue, Mém. ingénieur Ensia, Montpellier, France, 1999.

[15] Anonyme, Études pour la promotion des filières agro-industrielles. Vol. III. Analyse de l'état des filières des produits, Centre agro-entreprise (CAE), Étude Yiriwa Conseil, Bamako, Mali, février 2001. 


\section{El mango en África Occidental francófona: variedades y composición varietal de los huertos.}

Resumen - Introducción. El mango es uno de los árboles frutales más extendidos en África Occidental. Recientemente se publicó una reseña histórica de su introducción en la zona. Como continuación de dicho documento, este análisis está dedicado a las principales variedades que se cultivan actualmente y al impacto de los factores históricos y comerciales en la composición varietal de los huertos frutales. Algunas definiciones. Antes de inventariar las variedades, se especifican algunos términos que permiten caracterizarlas (precocidad, monoembrionía y poliembrionía) y se proporciona información sobre el origen de las variedades floridanas. Las principales variedades cultivadas en África Occidental. Se han distinguido cuatro categorías: las variedades de mangos locales o poliembriónicas (mangots, mango de Camerún), las primeras variedades monoembriónicas multiplicadas por injerto (Amélie, Julie, Sabot, Djibelor, Cuisse Madame), las variedades floridanas, también monoembriónicas y multiplicadas por injerto, introducidas más tardíamente y utilizadas, bien para la exportación (Kent, Keitt, Palmer, Zill, Valencia, Smith, Irwin, Haden), bien para los mercados regionales (Brooks, Davis-Haden, Miami Late, Springfels, Beverly, Eldon, Ruby). Se describió cada variedad, así como sus características de cultivo y salidas de mercado. Composición de las plantaciones frutales de mangos injertados. La composición de los huertos de mangos injertados está influida por la evolución histórica y el destino de la fruta que puede servir para autoconsumo, venta en el mercado local, nacional o subregional, exportación al mercado internacional o para su transformación en unidades artesanales o industriales. Se detalló dicha composición en Guinea, Malí, Burkina-Faso, Costa de Marfil, Senegal y Togo.

Africa de habla francesa / Mangífera índica / variedades naturalizadas / características agronómicas / precocidad / frutas / fenotipos

To access this journal online: www.edpsciences.org 